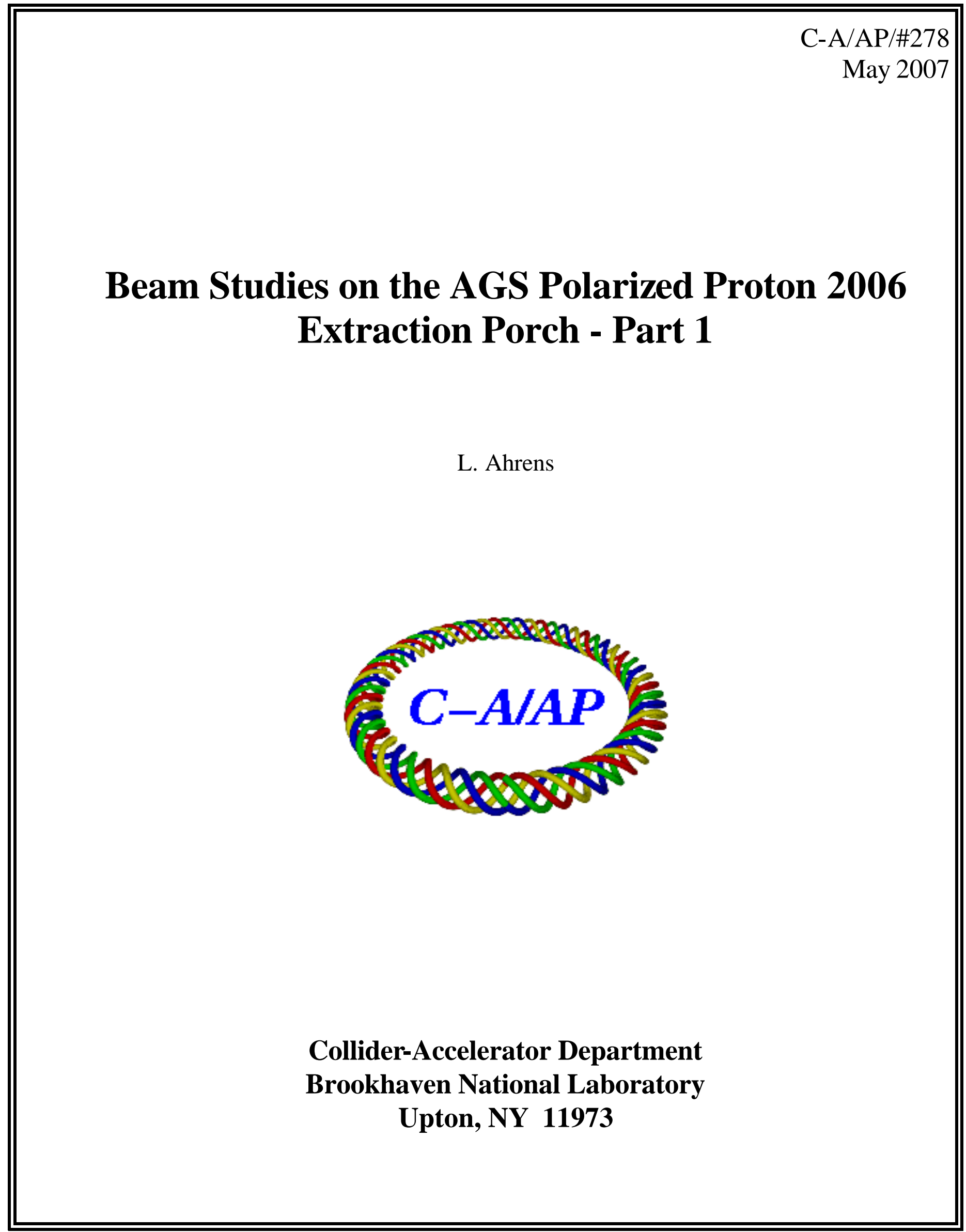




\section{Beam Studies on the AGS Polarized Proton 2006 Extraction Porch _ Part 1 \\ Ahrens May, 2007}

Contents:

Introduction and Summary

The Observation to be Explained

Dispersion at the IPM

Momentum shift with change in radial loop function

Conclusions

App I: Fitting the Momentum Distribution

App II: Flat top dispersion measurements and Model calculations

App III: Radial Loop Response

App IV: Aside on the bump, path length, and beam momentum

\section{Introduction and Summary}

Several measurements of beam parameters on the AGS extraction magnetic porch made during the 2006 polarized proton run are here reviewed. We learn a bit more about the machine conditions - relevant to understanding other behavior past and future. We also learn a bit about the instrumentation. The 'Part I' in the title indicates in particular that there is one major measurement - the ORM (Orbit Response Matrix) data - which is not covered here. To the extent that some shared instrumentation validation is demonstrated here, the efforts connect. These measurements were made during the second half of May. The raw data, or pointers to logged data are found in the BoosterAGS fy06-pp elog.

A particular observation made during the run initiated the present analysis. The horizontal emittance reported by the AGS IPM on the extraction flat top was observed to vary with time across the flat top with a minimum occurring just when the beam was extracted. The cause for the reported change was associated in time with the occurrence of the 'extraction bump' - the distortion of the horizontal equilibrium orbit to bring the circulating beam into the kicker magnet mouth and close to the extraction septum before the kicker magnet fires moving the beam across the septum and into the AtR line. This bump ramps on over $100 \mathrm{~ms}$, holds full value for 50ms and then ramps back down. The time dependence of the reported emittance followed this same time pattern. A semiquantitative explanation of the effect was provided during the run. The horizontal dispersion at the IPM is significantly reduced when the bump is up. The IPM does not correct the emittance that it reports to take into account momentum spread and the dispersion at the IPM. Even if it had, it would have been ignorant of the change in the dispersion caused by the bump. An attempt to push a quantitative revisit of this phenomenon was the initial and primary objective of this note.

In order to carry out this analysis, the dispersion at the IPM must be well known under both bump up and bump down conditions. (The bump follows a time dependent 
function. A "bump off" measurement can be made after the bump current has ramped down even though the power supply is on and pulsing or with the power supply in standby - just don't be confused. Here on/off refers to the presence of current at measurement time. Data to allow a measurement of dispersion - measurements of the equilibrium orbit and rf frequency for a set of radial loop settings - had been logged twice during May. In the course of digging out the dispersion some behavior of the radial loop was investigated, to come to a consistent picture of radius and frequency tracking. A parameter -namely a radial average measurement with a subset of pues (pick up electrodes) that lie within the bump excluded from that average - is introduced to help with this understanding. The hypothesis is that the average of horizontal pues excluding the set within the (inward -outward asymmetric) bump gives a measure of relative beam momentum to be compared with that obtained from the measured rf frequency change. This is further discussed in Appendix III.

The effect of the bump on momentum and path length are considered under two situations: the assumption of fixed frequency (normal condition for RHIC extraction) or fixed momentum (perhaps equivalent to running under the control of the radial loop). Comparing conditions (in time) at the center of the bump and after the bump is down, for running on the frequency loop or on the radial loop gives numbers for the frequency change and radius change consistent with the simple model that holds the magnetic field constant. This analysis implies that for frequency loop running ("synchro on"), the beam momentum at the moment of extraction is 0.05 units of G? lower than the momentum after the bump is down and where the AGS polarization measurement is taken

Having gone through these considerations, mainly in the appendices, the original objective - to quantitatively explain the observed behavior of the IPM during the bump is addressed. The bottom line from this is seen to not be entirely satisfactory. The beam momentum spread deduced from the IPM data is slightly but significantly larger than that measured by the conventional procedure - namely from the measured bunch length and rf voltage. 
The Observation to be Explained - two examples:

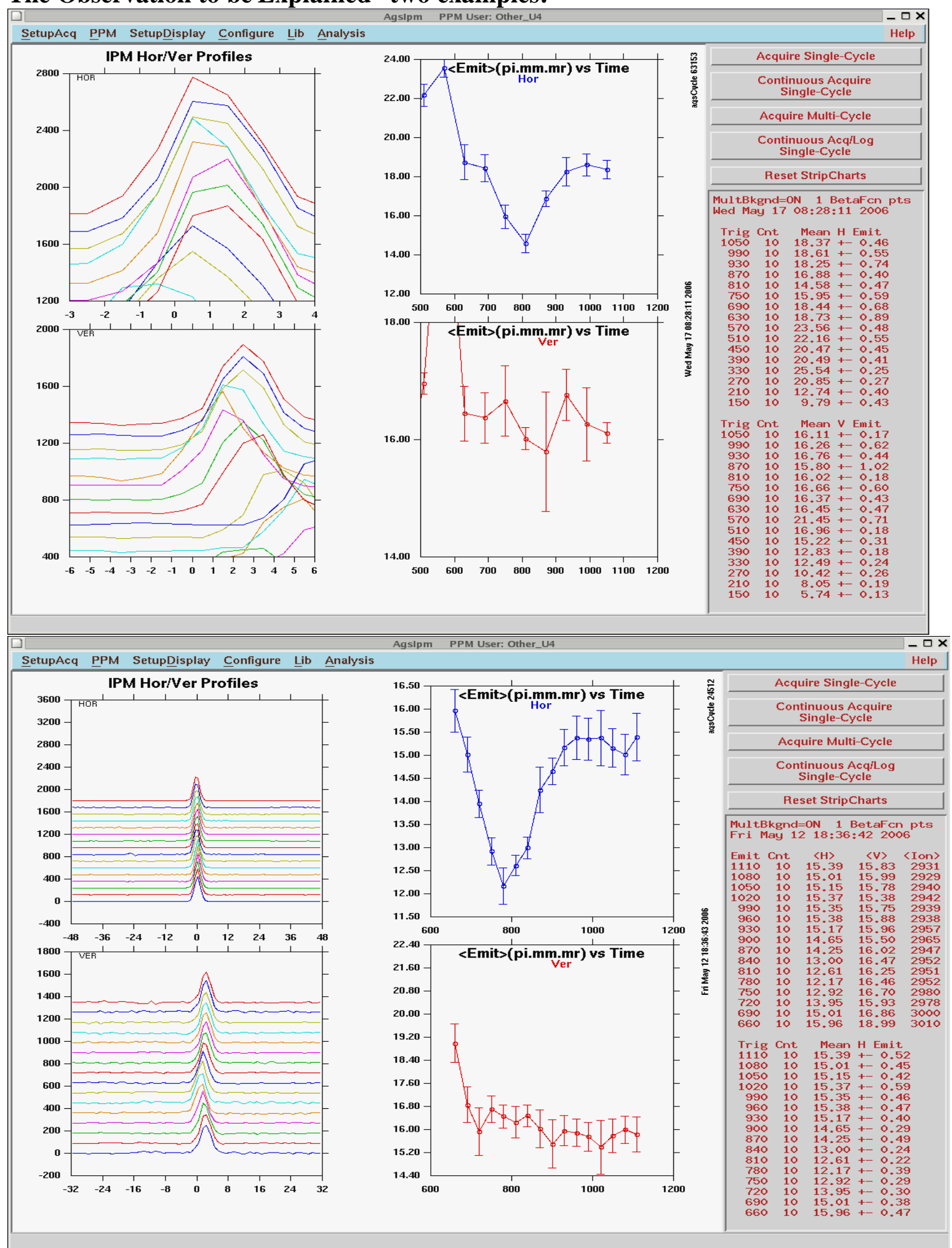

Figure 1 IPM response to the extraction bump 
The upper right graph in either picture of Figure 1 (the second picture is described specifically though either can be used - to give some idea of the effect and variations) displays the IPM's report of the normalized horizontal emittance as a function of time during the cycle starting at $600 \mathrm{~ms}$ - just as the beam arrives onto the extraction flat top. What one sees is a deep dip centered at 780-800 ms. and finished - recovered from - by $1000 \mathrm{~ms}$. In figure 1 the reported horizontal emittance goes from $12.2 \mathrm{pmmmr}$ at its lowest to $15.4 \mathrm{pmmmr}$ at the end of the trace. The emittance reported is calculated from a Gaussian fit to the profile collected. The sigma of that fit is translated into a $95 \%$ emittance:

Eq 1: $\quad \mathrm{e}_{\mathrm{n}}=\beta ?\left(6 \mathrm{~s}_{\mathrm{rms}}^{2}\right) / \beta_{\mathrm{t}}$

The reported emittance is the normalized emittance - hence the initial Lorentz factors beta and gamma. The sigma is found from fitting the beam profile to a Gaussian. The factor 6 turns the reported sigma from the rms emittance to the $95 \%$ emittance. The $\beta_{t}$ in the denominator is the lattice optics beta function evaluated at the IPM.

Usually what one wants to know from the IPM is the transverse emittance of the beam. That would be properly given by eq 1 provided the sigma were only that piece due to the transverse distribution of the beam:

Eq 2: $\quad s^{2}{ }_{\text {tot }}=s^{2}{ }_{\text {trans }}+\left(D_{\mathrm{x}} * d p / p\right)^{2}$

$D_{x}$ is the dispersion at the measurement point - the amount the beam moves for a given fractional change in its momentum (at a fixed main magnet field). The factor $\mathrm{dp} / \mathrm{p}$ is that fractional change, or in this case the momentum spread of the beam. The total beam size is assumed to result from the transverse motion and the momentum spread in an uncorrelated way, hence the combination in quadrature. The total sigma is assumed to be that of a Gaussian fit to the distribution. To get the same parameter for the momentum distribution, it too should be fit as well as possible to a Gaussian. See Appenxis I.

The model we are following then is to identify the dip in reported emittance with a change in the $D_{x}$ at the IPM. The other variables in the equation, $s^{2}$ trans and $d p / p$, are assumed not to change as the bump changes. The neat thing then is that if we know the dispersion and the two reported sigmas, bump on and off, we can solve for both the true transverse sigma and for the momentum spread.

\section{Dispersion at the IPM:}

The most straight forward way to learn what the dispersion is at the IPM is to measure how much the beam moves there when the beam momentum is changed by a known amount. The IPM can provide a centroid of the projection measured as well as a sigma.

Figure 2 gives a set of data taken 17May06 of this exercise. The centroids reported behave nicely linearly as the radial command is varied. The reported slopes are 
(-5.02(2) $\mathrm{mm} / \mathrm{V}$ and $-6.06(3) \mathrm{mmV}$ ) for (bump up and bump down) respectively. To turn this into dispersion values we need to know what one Volt of radial command is worth in terms of $\mathrm{dp} / \mathrm{p}$. That is the subject of the next section.

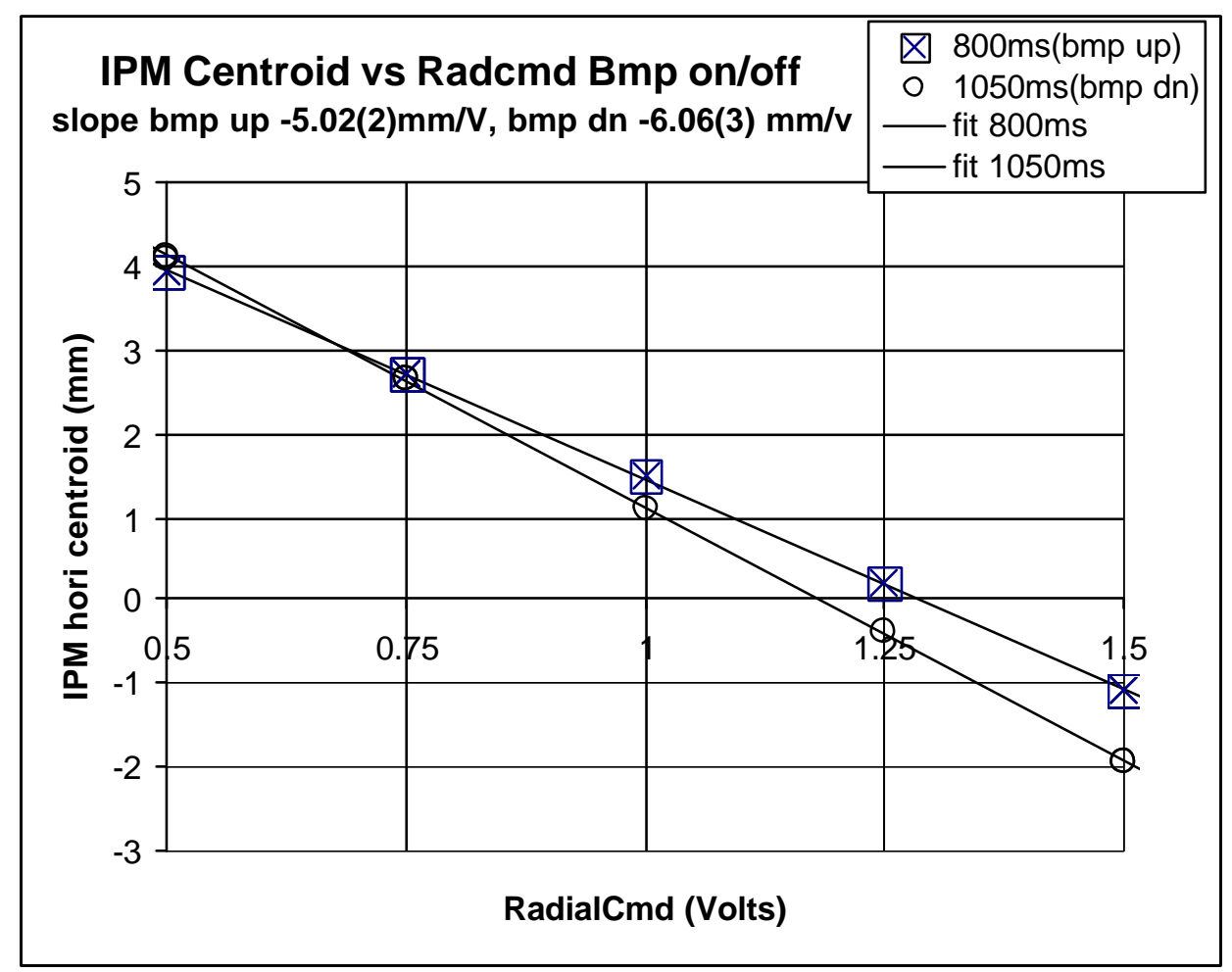

Figure 2 Change in IPM centroid with radial command, bump on/off

\section{Momentum shift with change in radial loop function}

The usual way to do this calibration is to measure by how much the rf frequency changes when the radial command is changed. The exercise is carried out at fixed magnetic field. This measurement was made 28May06. Figure 3 gives the results. The behavior of frequency vs radial command is well described as linear. One somewhat unusual aspect is that the answer depends on whether the bump is up or down. This we will rationalize about later (Appendix IV), but we continue.

We find slopes of $\{231(4) \mathrm{Hz} / \mathrm{V}$ or $5.19 \mathrm{e}-5 \mathrm{df} / \mathrm{f} / \mathrm{V}$ and $208 \mathrm{~Hz} / \mathrm{V}$ or $4.67 \mathrm{e}-5$ $\mathrm{df} / \mathrm{f} / \mathrm{V}$ \} for (bump up and bump down) respectively. ( $\mathrm{f}=4.453720 \mathrm{MHz}$ ). This translates (at fixed field and no funny business with path length while moving the radius, $\mathrm{dp} / \mathrm{p}=\mathrm{df} / \mathrm{f}$ $\mathrm{x}\left(?_{\operatorname{tr}}^{2} ?^{2} /\left(?_{\operatorname{tr}}^{2}-?^{2}\right)\right)$ into $3.8 \mathrm{e}-3 \mathrm{dp} / \mathrm{p} / \mathrm{V}$ bump off and $4.22 \mathrm{e}-3 / \mathrm{V}$ bump on. 


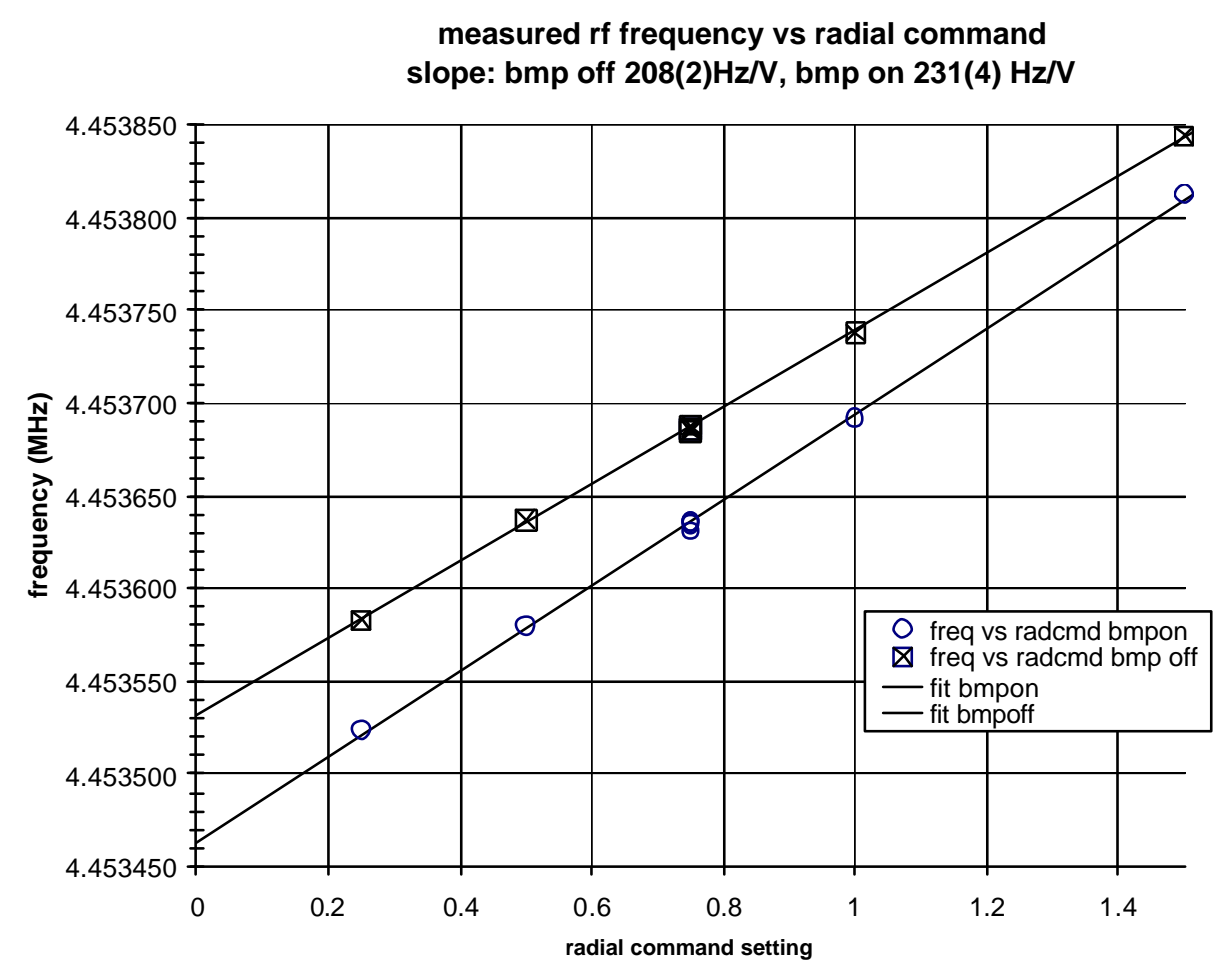

Figure3 Measured change in rf frequency with radial command bump on and off

Dispersion at the IPM : conclusion:

Combining the last two results, we learn the the position change at the horizontal IPM with momentum is: $(5.02 \mathrm{~mm} / \mathrm{V} \times 1 \mathrm{~V} / 4.22 \mathrm{e}-3)=\mathbf{1 . 1 9} \mathrm{m}$ with the bump up and $(6.06 \mathrm{~mm} / \mathrm{v} \times 1 \mathrm{~V} / 3.8 \mathrm{e}-3)=\mathbf{1 . 5 9} \mathbf{m}$ with the bump down. These are the dispersion values required.

A comparison of these values with the dispersion measured at the ring pues and to a model is given in Appendix II. The picture is consistent.

Armed with these results, we can return to eq 2 (restated below) and given $\mathrm{s}^{2}$ tot for the bump up and bump down cases, and the two dispersions, solve for $\mathrm{dp} / \mathrm{p}$ and $\mathrm{s}^{2}$ trans.

Eq 2: $\quad s^{2}{ }_{\text {tot }}=s^{2}{ }_{\text {trans }}+\left(D_{x} * d p / p\right)^{2}$

Then $(1=$ off, $2=$ on $)$

Eq3: $(\mathrm{dp} / \mathrm{p})^{2}=\left(\mathrm{s}^{2}{ }_{\text {tot } 1}-\mathrm{s}^{2}{ }_{\text {tot2 } 2}\right) /\left(\mathrm{D}_{\mathrm{x} 1}{ }^{2}-\mathrm{D}_{\mathrm{x} 2}{ }^{2}\right)=\left(? \mathrm{~s}_{\text {tot }} / ? \mathrm{D}_{\mathrm{x}}\right) \mathrm{x}\left(\left\langle\mathrm{s}_{\text {tot }}\right\rangle /\left\langle\mathrm{D}_{\mathrm{x}}\right\rangle\right)$

and

Eq 4: $s_{\text {trans }}^{2}=\left(D_{\mathrm{x} 1}^{2} s_{\text {tot2 }}^{2}-D_{x 2}{ }^{2} s_{\text {tot } 1}^{2}\right) /\left(D_{\mathrm{x} 1}^{2}-D_{x 2}{ }^{2}\right)$ 
To get sigmas from the IPM to plug into this expression, and given that what is saved is normalized emittance, we must undo the effort of the application by equation 1 .

$$
\text { Repeat of Eq 1: } \quad \mathrm{e}_{\mathrm{n}}=\beta ?\left\{6.25 \mathrm{~s}_{\mathrm{rms}}{ }^{2}\right) / \beta_{\mathrm{t}} \quad \text { (yes the code uses the factor 6.25) }
$$

For standard data taking the IPM assigned a $\beta_{\mathrm{t}}$ of 22 meters to the IPM. 3 ? at extraction is $\sim 25.4$. Then plugging the emittance numbers from figure 1 (off: $15.3 \mathrm{p}$ $\mathrm{mmmr}$, on: $12.4 \mathrm{p} \mathrm{mmmr}$ ) gives $\mathrm{dp} / \mathrm{p}=0.6 \mathrm{e}-3$ (1.41e-3 full amplitude) and (sticking with the same 22 meter beta function at the IPM an emittance of $8.8 \mathrm{p} \mathrm{mmmr}$.

As was mentioned in the introduction and Appendix I, typical longitudinal rms emittance widths tend to be slightly larger than what is typically calculated using the wall monitor and rf gap voltage. In this particular case the FWHM required would be 16 ns whereas we measure $15 \mathrm{~ns}$. $15 \mathrm{~ns}$ would correspond to an rms dp/p of $0.57 \mathrm{e}-3$ and a full $\mathrm{dp} / \mathrm{p}$ amplitude of $1.13 \mathrm{e}-3$. Table 1 below gives a small sampling of the many measurements taken during the run. Oddly, early in the run the dip is frequently not obvious.

The last equality in Eq 4 above, the expression for dp/p in terms of the changes in sigma and dispersion (bump on and off), being proportional to one on the difference in dispersions indicates how sensitive the answer is to having correct dispersion values as the change in dispersion gets small.

\begin{tabular}{|l|l|l||l|l|l|}
\hline date & $\begin{array}{l}\text { e }(\mathrm{p} \mathrm{mm} \mathrm{mr}) \\
\text { norm 95\% } \\
810 \mathrm{~ms}\end{array}$ & $\begin{array}{l}\text { e (p mmmr) } \\
\text { norm 95\% } \\
<630 \mathrm{~ms}, 1000 \mathrm{~ms}>\end{array}$ & $\begin{array}{l}\text { e trans }(\mathrm{p} \\
\text { mmmr } \\
\text { norm 95\% }\end{array}$ & $\begin{array}{l}\text { rms } \\
\mathrm{dp} / \mathrm{p} \\
\mathrm{e}-3\end{array}$ & $\begin{array}{l}\text { full } \\
\mathrm{dp} / \mathrm{p} \\
\mathrm{e}-3\end{array}$ \\
\cline { 2 - 6 } 2May & 11.76 & 14.45 & 8.38 & 0.57 & 1.34 \\
\hline 2May & 10.60 & 12.9 & 7.71 & 0.53 & 1.25 \\
\hline 2May & 10.96 & 13.1 & 8.27 & 0.51 & 1.21 \\
\hline 3May & 11.88 & 14.2 & 7.98 & 0.53 & 1.25 \\
\hline 3May & 10.33 & 12.2 & & 0.48 & 1.13 \\
\hline $\begin{array}{l}\text { from rhic } \\
\text { elog: }\end{array}$ & & & 7.65 & & \\
\hline 5May & 10.63 & 13 & 8.15 & 0.49 & 1.27 \\
\hline 5May & 10.57 & 12.5 & 7.95 & 0.46 & 1.08 \\
\hline 7May & 10.15 & 11.9 & 8.42 & 0.62 & 1.46 \\
\hline 12May & 12.36 & 15.5 & 7.96 & 0.62 & 1.46 \\
\hline 16May & 11.88 & 15 & 8.11 & 0.54 & 1.27 \\
\hline 18May & 11.11 & 13.5 & 12.03 & 0.47 & 1.11 \\
\hline 10Jun & 14.24 & 16 & &
\end{tabular}

Table 1: Bump on/off "raw" emittance measurements and extracted emittance and momentum spread 


\section{Appendix I Fitting the Momentum Distribution}

The normal way the momentum distribution of the beam in the AGS is obtained is by measuring the response of the AGS "wall monitor". This device gives a high fidelity representation of the time dependence of the beam intensity at a point in the AGS ring as the bunch passes. In the AGS the monitor is located in the G5 straight section. Its signal is sent to MCR console3 by a short low-distortion coaxial cable.

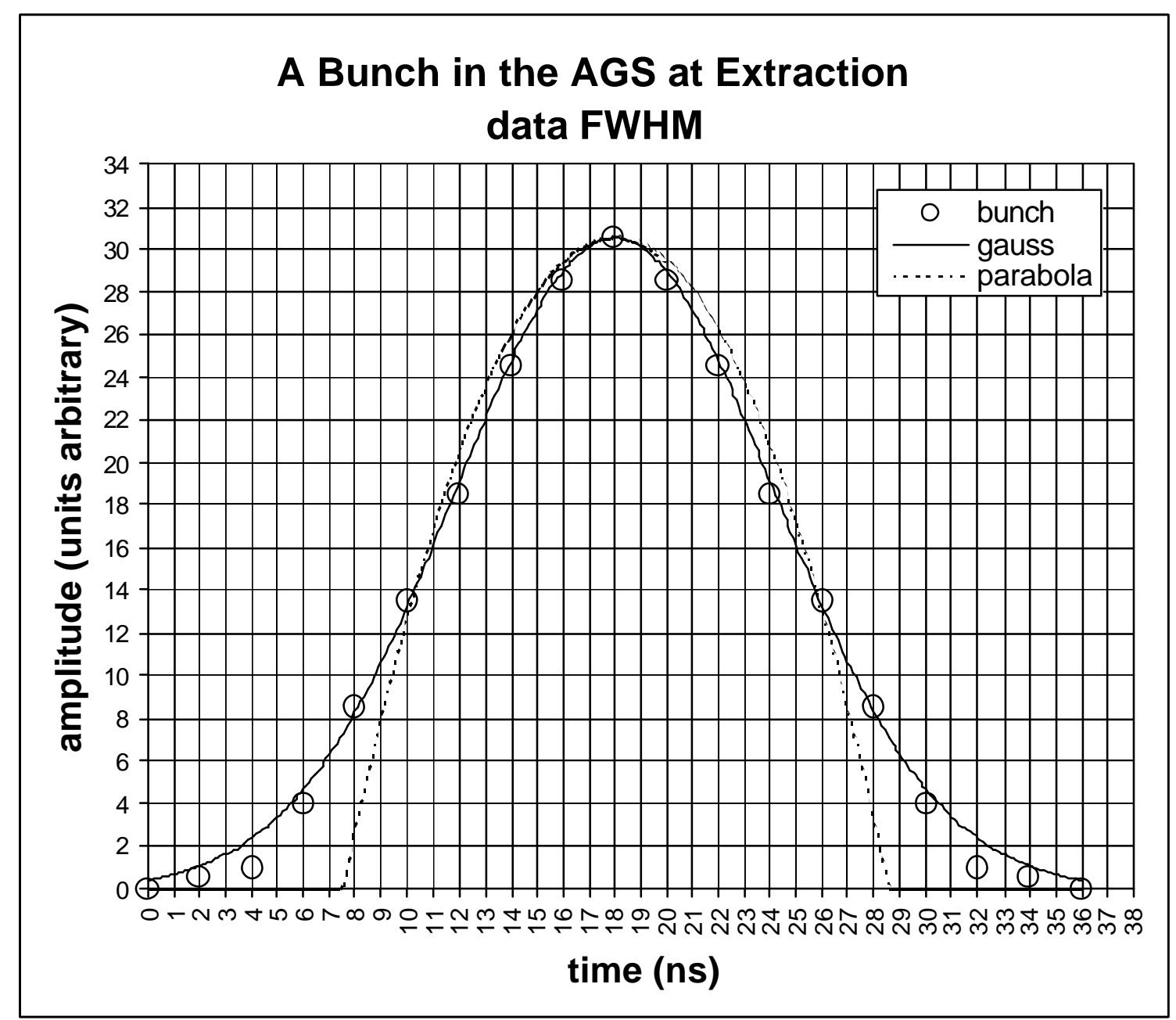

Figure AI.1 Longitudinal bunch at AGS Extraction

The dots in the figure are taken from one particular measurement of the bunch. The claim is this is adequately "typical" for the present analysis. The Full Width at Half Max (FWHM) of this data is about $15 \mathrm{~ns}$. The solid line is an overlayed Gaussian function. It's rms sigma is $6.2 \mathrm{~ns}$. The Gaussian and the data overlay well except out on the tails where the data cuts off more quickly that the function. Also shown is a parabolic function (dotted) which is a poor representation of the data. For a Gaussian the FWHM is equal to the rms sigma $\mathrm{x}(\mathrm{k})$ where $\mathrm{k}=2.35$. This is taken as a reasonable way to connect the measured bunch lengths and the momentum spread, namely the measured FWHM is 
to be divided by $\mathrm{k}$ to get the appropriate sigma in combining momentum spread with inherent transverse spread.

Ok, we are not mentioning a critical piece in this analysis. To the extent that the following digression is true, it is not original. The "momentum spread" measurement is really a measurement of the bunch width in time. This is the projection of the bunch distribution ( a distribution in the longitudinal phase space with axes time deviation and momentum deviation from the central - synchronous - particle) onto the time axis. What we want is the projection onto the momentum axis. The particles in the distribution are constantly moving around the center at (approximately - the bunch is small compared with the bucket length, only the particle infinitesimally away from the central particle moves exactly at the synchrotron frequency) the synchrotron frequency - about $150 \mathrm{~Hz}$ at AGS extraction, so once around every $\sim 7 \mathrm{~ms}$. The observed time projection nevertheless does not change with time - well nearly. If we could see the momentum projection directly it too would not change with time. Following a particular particle around its trajectory in this space, we can find its maximum time excursion ( momentum deviation zero) and after a quarter synchrotron oscillation travel its maximum momentum excursion (now centered in time in the bunch). If we do this for particles with different max times, we will find that the max time and the associated max momentum keep a constant ratio. We can define our units to make the motion in this phase space look like circles. So now the distributions in time and momentum are the same. We can calculate the momentum deviation associated with a given time offset. The rf group generated programs Bbat or Bbrat are an easy way to get the numbers for our situation. Giving that program the width of the bunch - twice the max excursion of our particle from the centerthe rf voltage the beam can see per turn - learned from the measurable synchrotron frequency, again using the Bbat tool - and other AGS parameters, the program returns the associated momentum offset for that particle. The particle with rms time offset has the rms momentum offset.

In the particular example above, these are the numbers. The synchrotron frequency was measured to be $165 \mathrm{kHz}$. The rf voltage required to make this frequency is $150 \mathrm{kV}$. A particle with a 30ns full time excursion has dp/p of $1.14 \mathrm{e}-3$. (This is about the "full width at the base" for our typical bunch, and is the sort of number for the momentum spread usually quoted). For a particle at the FWHM time, dp/p would be half that, .57e- 3 , and the rms guy would be $0.49 \mathrm{e}-3$.

Going the other way, if we start with an rms dp/p of $0.7 \mathrm{e}-3$, the 'usual' number has to grow to $1.63 \mathrm{e}-3$ and a base width of over $40 \mathrm{~ns}$.

\section{Appendix II:}

\section{Flat Top Dispersion Measurements and Comparison to Model Calculations}


The modeling of the ags machine dispersion at extraction displayed in this section was done by N. Tsoupas. Careful consideration of how the ags backleg bumps are introduced into the "standard" mad model is necessary to get a consistent answer as the various parameters are varied. This modelexperiment cross-checking is probably even necessary to keep both approaches on the reality plane.

Several sets of orbits vs momentum were taken during May. These data can be translated into dispersion values at the pues. Figure AII 1 gives the results for the bump on case. Two independent scans are shown (open circles and black dots. The error bars on the circles indicate the variation in the orbit data over several consecutive samples. What is displayed is a difference between a momentum shift outward and inward. The calibration of the radial loop reference function to learn associated momentum shift is discussed in the main part of this note. As indicated there, different calibrations are necessary for the bump on data and for the bump off data.

Also plotted in the figure is a model prediction for this situation. Here the settings for the quadrupole and sextupole strings are taken from the running machine. The bumps are modeled by changing the current in the appropriate combined function magnets, i.e. realistically. This is necessary to get a reasonable agreement on the dispersion. (A very similar plot was included in the Jun 4 pp elog only with the bumps modeled by additional point dipoles. The resulting dispersion function had significantly larger excursions.
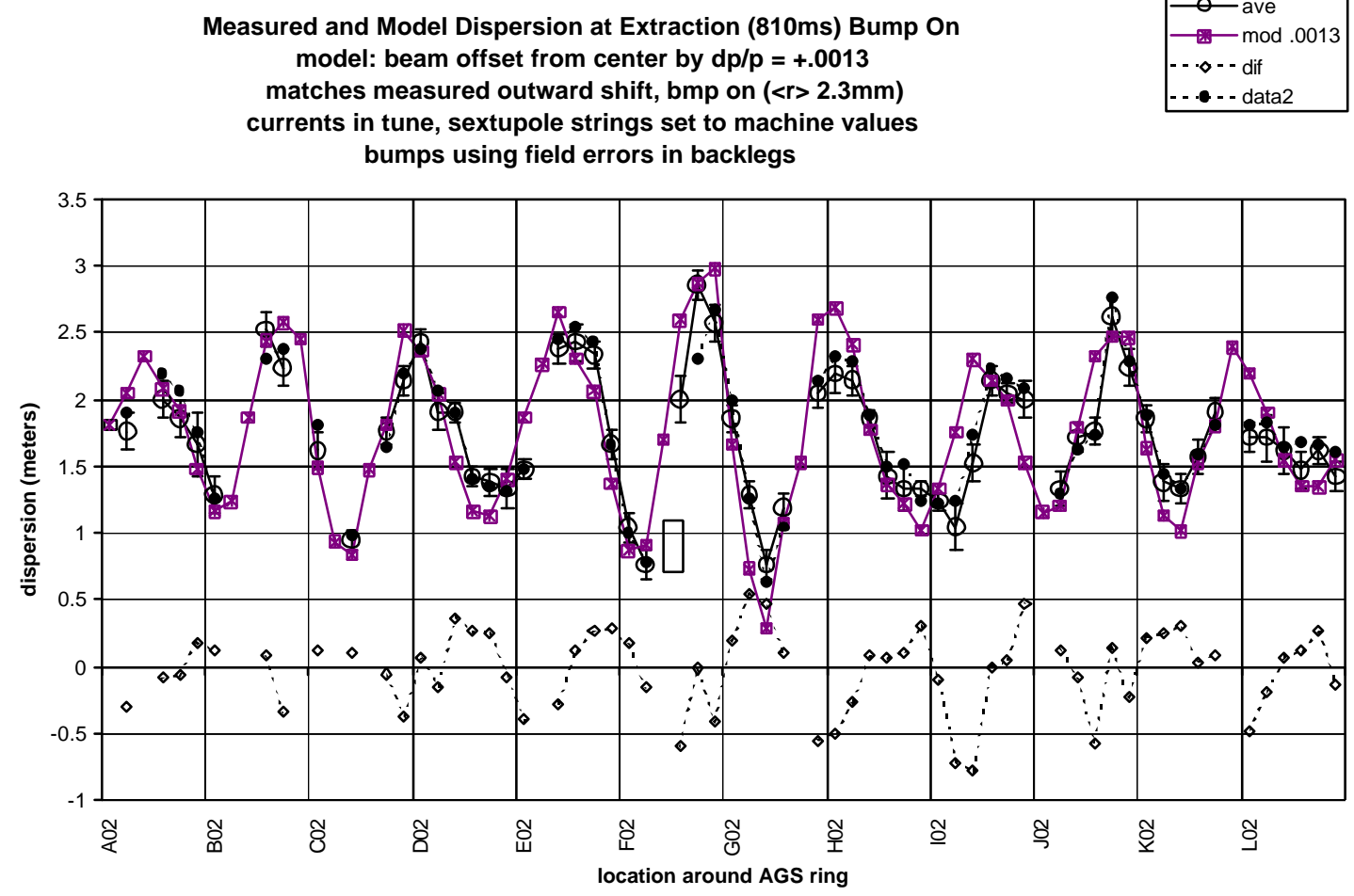

Figure AII 1 Measured and calculated dispersion, bump on. 
Also shown in the figure is the difference between the experimental data and the model. The difference has a smooth component indicating that we do not yet have the model completely right. The model prediction at C5 is $0.992 \mathrm{~m}$ compared to our extracted value of $1.19 \mathrm{~m}$. The model is also below the orbit-derived dispersion function at the adjacent pues - by 0.17 at $\mathrm{C} 2$ and 0.13 at $\mathrm{C} 8$. We take this as reasonable agreement.

Figure AII 1 gives a similar comparison for the bump off case. The agreement (beam vs model) is excellent - well within the pue data error bars..

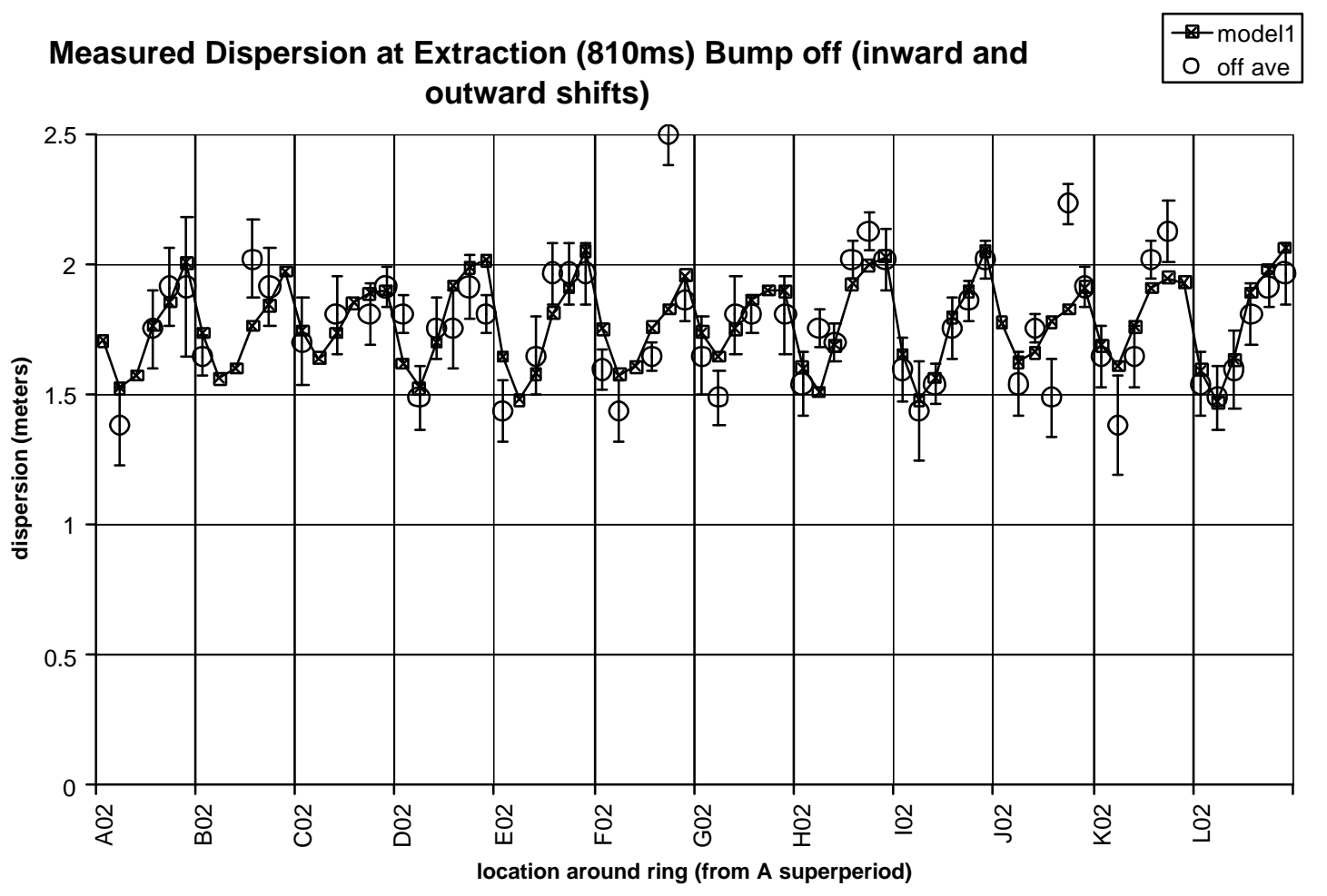

Figure AII 2 Dispersion bump off, orbit data and model

\section{Appendix III: Radial Loop Response}

As mentioned in the main report, the response of the radial loop produces a momentum shift dp/p of $3.8 \mathrm{e}-3 / \mathrm{V}$ when the bump is down and $4.22 \mathrm{e}-3 / \mathrm{V}$ when the bump is up. These numbers are extracted from the measured frequency changes with radial command change, and assume the only path length changing during each radial scan is associated with the momentum change. To explore this a bit further, a second measure of momentum, namely the average of a subset of the horizontal pues, is introduced. This is labeled $\langle\mathrm{r}>\mathrm{p}$. This radial measure gives a number proportional to the momentum change (at fixed field) through the momentum compaction factor. Although the bump itself 
causes a first order path length change with amplitude, the expectation is that symmetric inward and outward bump residuals will result in path length changes that can be neglected. Therefore considering an average of pues away from the bump should give a number proportional to the momentum shifts. The cut is made by excluding pues from F8 through $\mathrm{J} 2$.

As a "necessary" condition for the correct behavior of this momentum parameter $(<r>p)$, it must give about the same number for the average as that extracted from the full set of pues $(<r>$ all $)$ when the bump is off. An orbit data set (29Apr06, sunchro on) took orbits on the bump and after the bump (828ms and $1153 \mathrm{~ms})$. Evaluating $\langle\mathrm{r}\rangle$ all and $\langle\mathrm{r}\rangle \mathrm{p}$ we get table 1 . The shift $(0.5 \mathrm{~mm})$ seen between the two bump off evaluations (which ideally would be zero and which is probably due to the granularity of the pue sets) would correspond to a momentum shift of $2.5 \mathrm{e}-5$, an acceptable error.

\begin{tabular}{|l|l|l|}
\hline $\begin{array}{c}\text { momentum } \\
\text { parameter }\end{array}$ & bmp on (828ms) & bmp off (1153ms) \\
\hline$<\mathrm{r}>$ all & $3.53 \mathrm{~mm}$ & $4.54 \mathrm{~mm}$ \\
\hline$<\mathrm{r}>\mathrm{p}$ & $2.51 \mathrm{~mm}$ & $4.59 \mathrm{~mm}$ \\
\hline
\end{tabular}

Table 1 Orbit Based Momentum Parameter Test

The next two figures show how this $<\mathrm{r}>\mathrm{p}$ measure behaves vs radial command bump on and off (figure 1), and when plotted against frequency, bump on and off (figure 2).

bpm average (corr, exclude bump region) vs radial command slopes: $-7.48(6) \mathrm{mm} / \mathrm{V}$ bmp on $-6.85(3) \mathrm{mm} / \mathrm{V} \mathrm{bmp}$ off

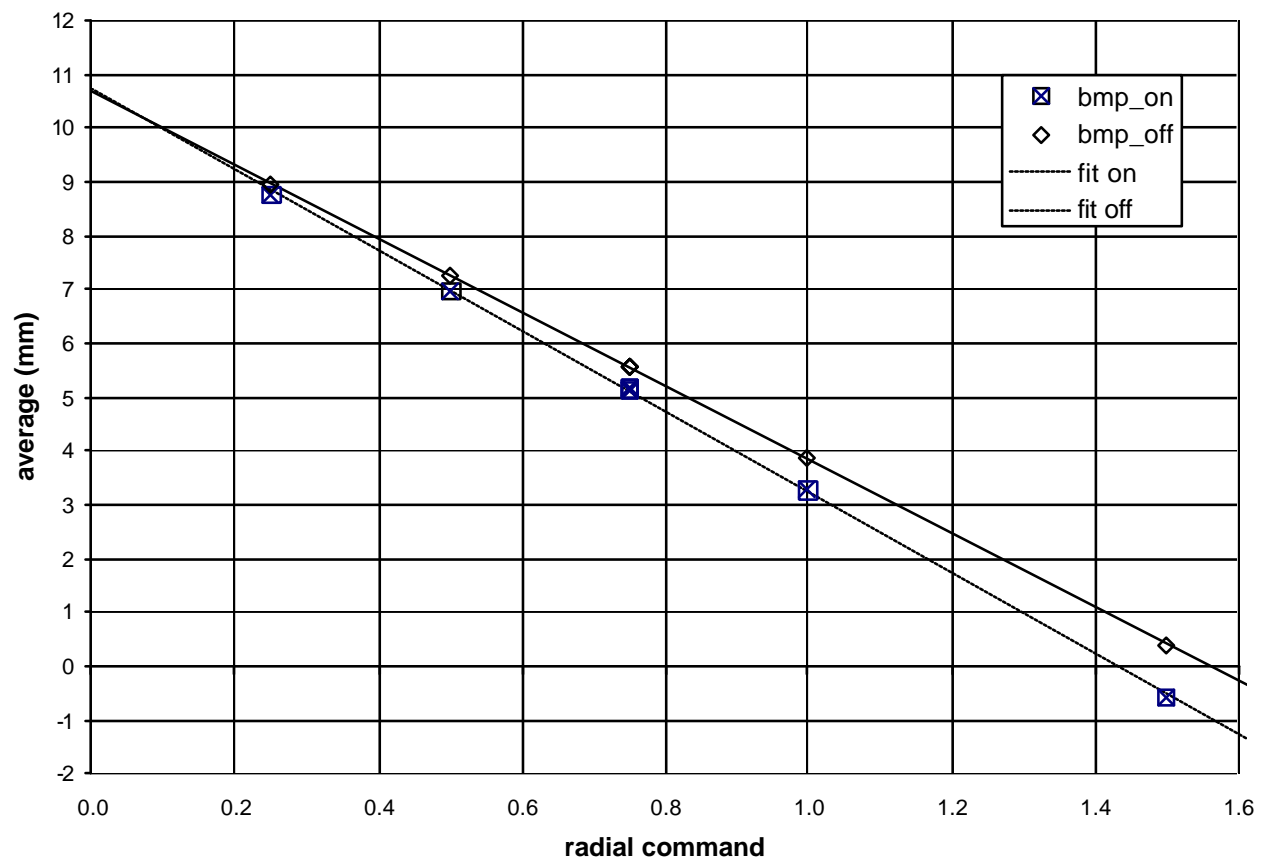

AIII Figure 1: $<r>p$ (pue average excluding those within the bump) vs radial command 


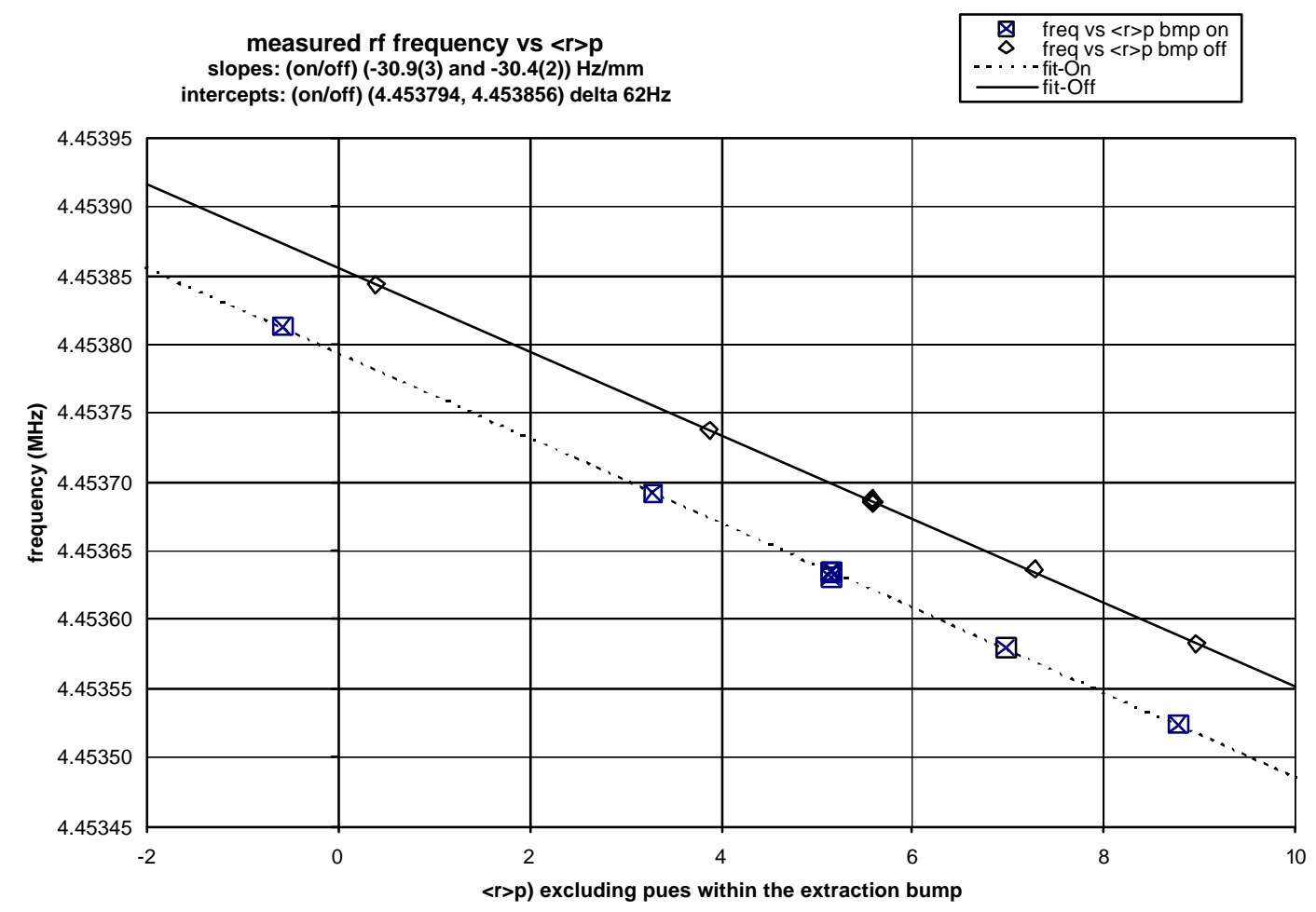

AIII Figure 2: Measured frequency vs $<\mathrm{r}>\mathrm{p}$. That these are parallel lines indicates they are consistent in their interpretation of the beam momentum. The frequency difference (at fixed $\langle\mathrm{r}>\mathrm{p}$ is $62 \mathrm{~Hz}$, the predicted effect for an $11 \mathrm{~mm}$ bump introduced at fixed momentum.

Why should the radial loop be affected by the bump? The loop uses two pues sets, located at B18 and C12 to learn the beam position and then shifts the momentum until the measured average signal from those pues is equal to the requested value - the radial function. These pues are chosen because the betatron phase advance between them is nearly 180 degrees. Adding a bump residual - which oscillates as a betatron oscillation provided the sourse is not between the pue locations - will to first order move the beam equal but opposite amounts at the two pues with no requirement that the loop do anything. Also, adding a dispersion wave to the machine, which again looks like a betatron oscillation, will not change the average dispersion at the two pues. So again to first order if the system is initially holding the beam at a certain dp/p away from the central momentum and with the same dispersions at both pues, then changing those dispersions one up and one down will not change the average and hence require the loop to do nothing. The bump off situation is a machine whose equilibrium orbit at the two radial pues is measured to differs by about $5 \mathrm{~mm}$ on the flat top. The dispersions (from the above mentioned model) are $1.97 \mathrm{~m}$ and $1.85 \mathrm{~m}$ at B18 and C12 respectively. The same numbers when the bump is up are $19 \mathrm{~mm}$ equilibrium orbit difference between the pues due to the bump residuals (whose peaks fall nearly at the pues) and model dispersions now $1.48 \mathrm{~m}$ and $2.45 \mathrm{~m}$ (again by chance well away from the possible phase near-null). If the model is taken at face value, there is a change in the average dispersion at the pues of 
about $2.5 \%$, greater with the bump on. Since the measured slope change is about $10 \%$ this is not the full story. If the gains of the electronics for the two pue locations were different, this would also contribute to a slope change. The effect is proportional to the shift of the pue dispersion away from the average dispersion, which is (using the model numbers) a $25 \%$ effect, so a $10 \%$ gain difference of the right polarity would contribute another $2.5 \%$.

\section{Appendix IV: Aside on the bump, path length, and beam momentum}

During polarized proton running beam behavior at the start of the flat top is complicated. The momentum (or G?) excursions forced on the beam are relevant to understanding the polarization of the beam sent to RHIC or measured in AGS on the subsequent flat top. (Indeed one unsolved mys tery from the 06 run was the apparent dependence of the polarization on the state (on or off) of the extraction bump, where the curiosity was that having the bump powered improved the measured polarization.)

\section{Description of the extraction setup:}

We should say that the extraction setup was seriously reworked at the end of March. The ramp up of the bump was moved later to coincide with the beginning of the synchro exercise. The radial shift timing and magnitude going into synchro was adjusted. The description here deals with the setup following that change, looking after April 1. It is believed that the longitudinal extraction setup remained nearly constant for the rest of the run, with some minor modification in the radial function on 17 May.

The beam is accelerated onto the AGS flat top. The main magnet power supply shifts from the accelerating "bank" over onto the flat top bank and settles down to holding a fixed current in the magnets as best it can. The radius is shifted outward in preparation for getting to the momentum required by RHIC or perhaps to optimize the functioning of the synchro loop. The extraction bump starts to ramp up and the synchroto RHIC rf gymnastics commence. All this is happening together and concludes shortly before the kicker fires and the bunch is extracted (FebReq 790ms, extraction $\sim 818 \mathrm{~ms}$ ). Then the bump ramps back down. This is the 'extract to RHIC' scenario. Other setups sometimes documented include staying on the radial loop throughout the flat top. And we have data with the bump powered and not powered.

First we look at the situation on the radial loop. The top trace in figure 1 is the radius. The sharp drop starting at $685 \mathrm{~ms}$ is the function request. The apparent overshoot at $700 \mathrm{~ms}$ is not understood. The small soft shift starting at $\sim 880 \mathrm{~ms}$ is again the function sent to the loop. The lowest trace shows the Main magnet power supply's voltage and above that the current. Flat top starts at about $600 \mathrm{~ms}$ - one box in.. 

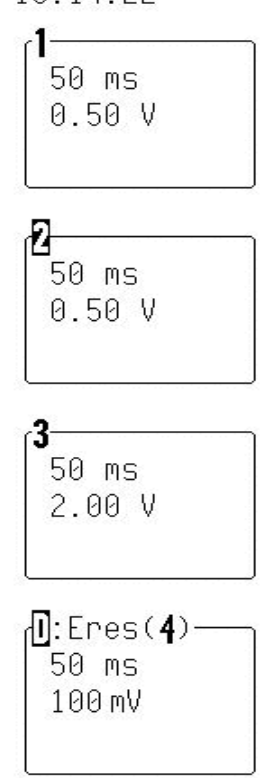

50 ms

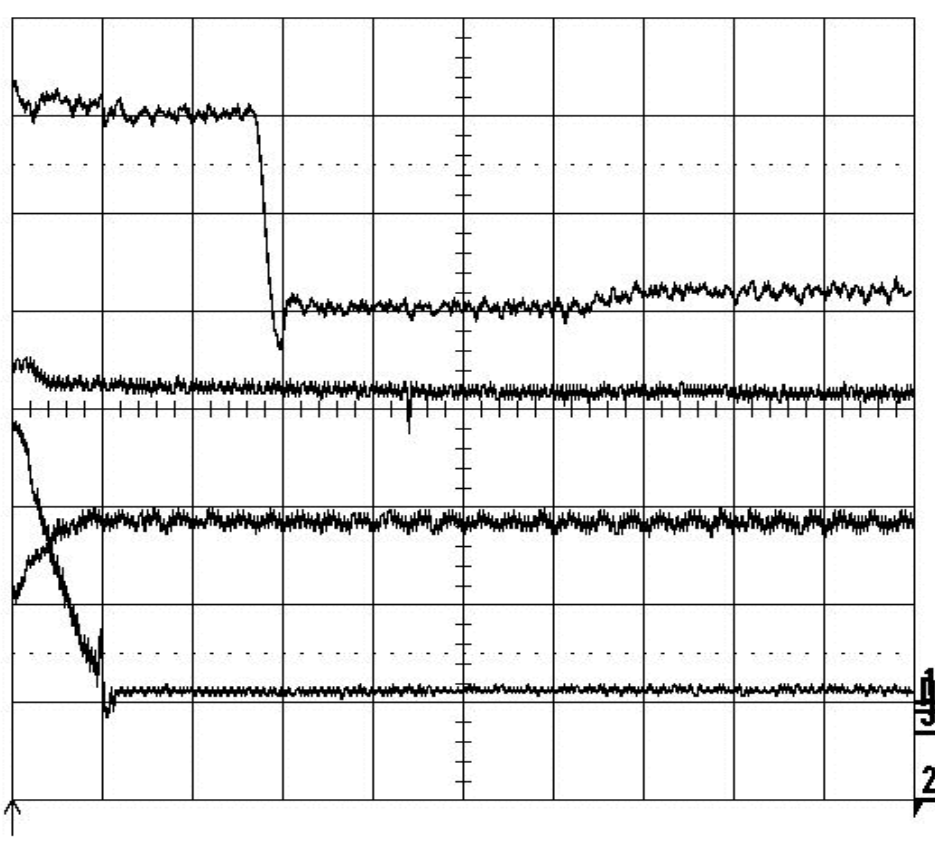

GO TO

LOCAL

$\begin{array}{rrrr}\mathbf{1} & .5 & V & D C \\ \mathbf{2} & .5 & V & D C \\ \mathbf{3} & 2 & V & D C \\ \mathbf{4} & .1 & V & D C\end{array}$

Figure AIII 1: scope trace of radius, intensity, Main Magnet voltage and Main Magnet current. Trigger at $550 \mathrm{~ms}$ T0.

What is the time structure of the bump? Traces of the current in one of the power supplies are shown in figures $2 \mathrm{a}$ and $\mathrm{b}$. The first was taken 17May the second 6 Apr. This bump supply ramps up starting at $680 \mathrm{~ms}$, is up by $780 \mathrm{~ms}$, holds flat till $820 \mathrm{~ms}$ and then ramps down by $930 \mathrm{~ms}$.

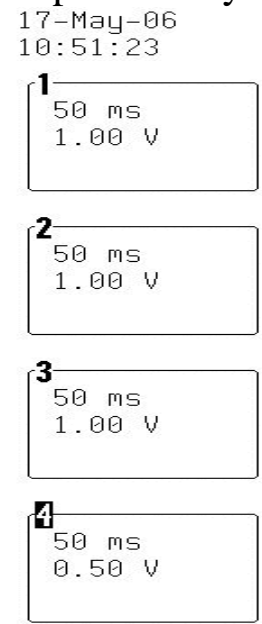

$\begin{array}{llll}50 & m s & & \\ \mathbf{1} & 1 & v & D C \\ \mathbf{2} & 1 & v & D C \\ \mathbf{3} & 1 & v & D C \\ \mathbf{4} & .5 & v & D C\end{array}$

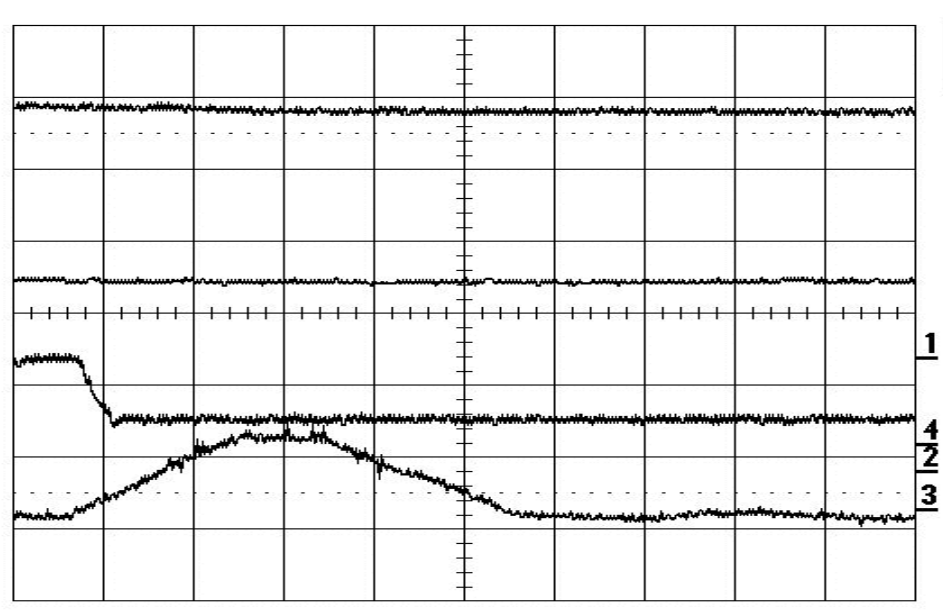

REMOTE ENABLE

$10: 51: 23$

$\left[\begin{array}{l}50 \mathrm{~ms} \\ 1.00\end{array}\right.$

$\longleftarrow 650$ ms

$100 \mathrm{kS} / \mathrm{s}$

Ext DC $225 \mathrm{mV} 5 \mathrm{A \Omega}$

$\square$ NORMAL 
Figure AIII 2a: intensity, rf volts, radius, and extraction bump. Trigger ags T0 21May

6-Apr-B6

$18: 47: 00$
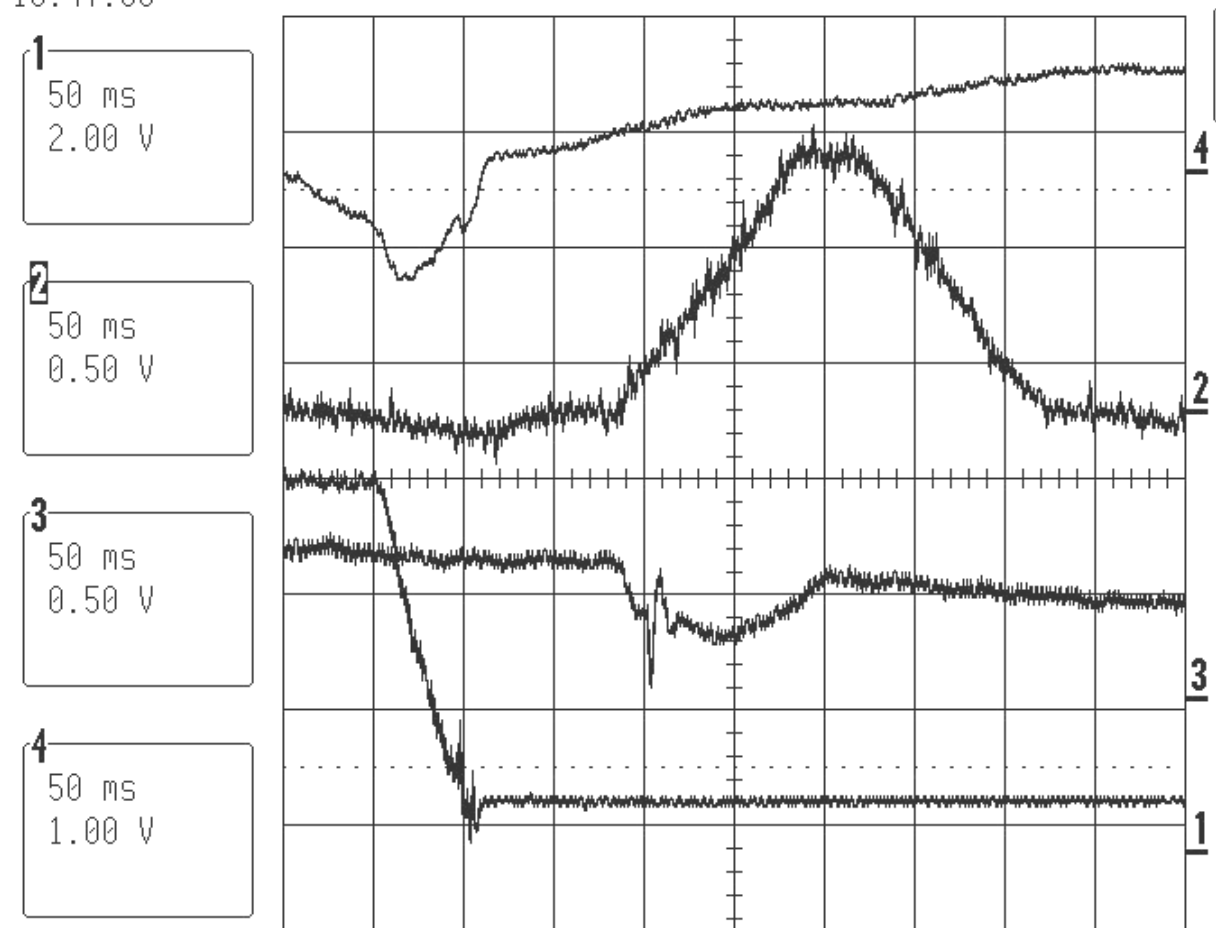

REMOTE ENABLE

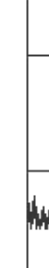

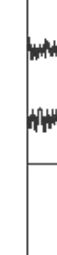

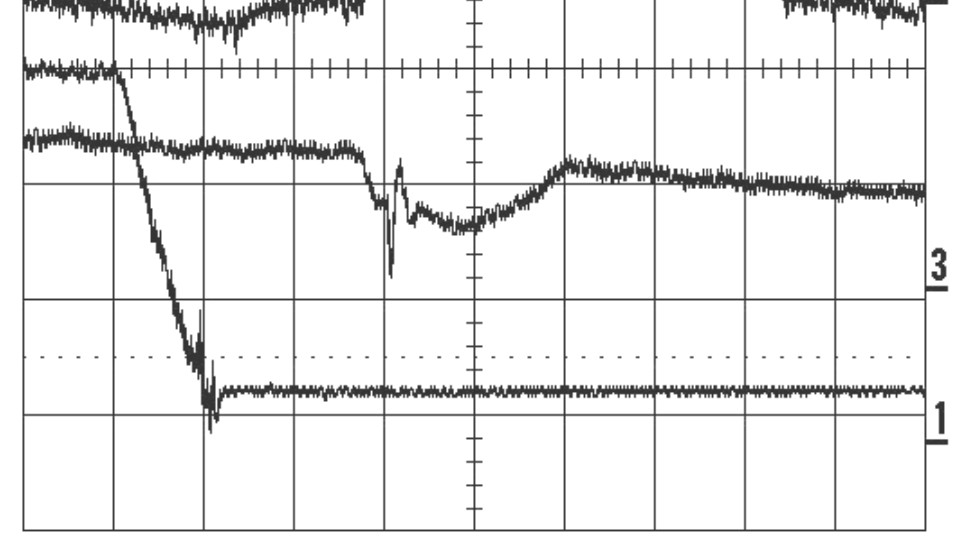

$\begin{array}{llll}50 & m 5 & \\ 1 & 2 & V & D C \\ 4 & .5 & V & D C \\ 3 & .5 & V & D C \\ 4 & 1 & V & D C\end{array}$

$\longleftarrow 500$ MS

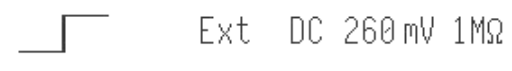

$100 \mathrm{KS} / \mathrm{s}$

SLOW TRIGGER

口 NORMAL

Figure AIII 2b: AGS main magnet power supply current error, bump current, radius, and main magnet voltage. The trace starts $500 \mathrm{~ms}$ after To.

Now some pictures of rf frequency over this time interval are presented. Figure 3 shows the beam frequency starting before the beam has reached flat top - hence ramping up vigorously.. Here the structure seen can be identified first with coming onto the flat which included a spike, then a 50 ms nearly flat period on the flat top before any gymnastics. Then a small decrease followed by a sharp downward step. The latter corresponds to the radial function request. The radial function is flat (at $1.3 \mathrm{~V}$ ) till $685 \mathrm{~ms}$ where in $10 \mathrm{~ms}$ it drops to $0.93 \mathrm{~V}$, which it holds till $862 \mathrm{~ms}$ at which time it starts to ramp back to 0.96 at $900 \mathrm{~ms}$. Superimposed on this radial function there is a smooth down and up associated with the bump. 


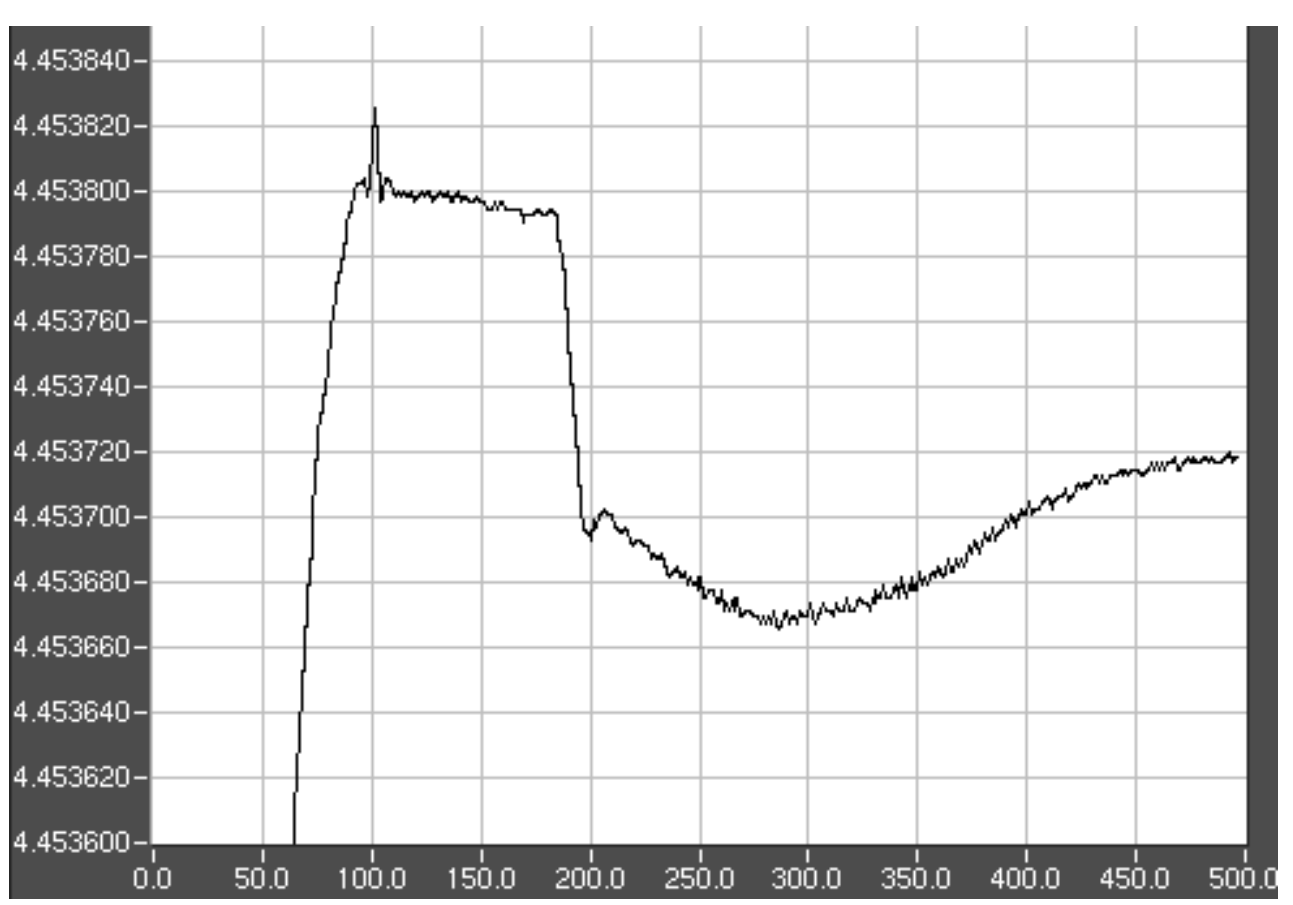

Figure AIII 3a: RF Freq over extraction flat top start, on radial loop, trigger 500ms T0, 50ms/box, 20Hz/box 3Apr06

Another similar frequency measurement is given in figure 4 . This was taken near the end of June. The radial function is a bit simpler than described above shifting out from 1.3 at $685 \mathrm{~ms}$ to 0.75 at $705 \mathrm{~ms}$ and staying there. The trace has the time dependence of the bump and a depth of about $50 \mathrm{~Hz}$.

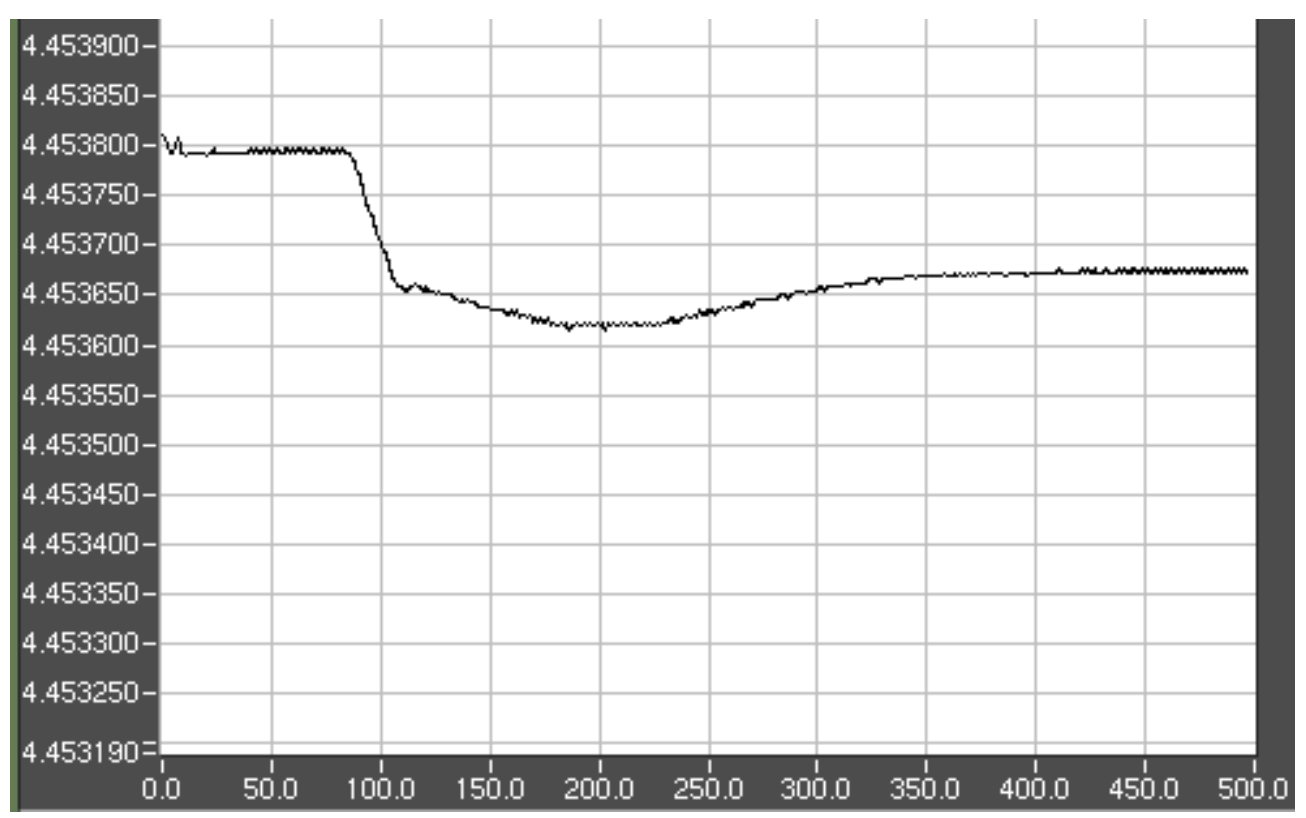

Figure AIII 3b: Beam frequency, on radial loop 21 Jun 06 
These figures give the experimental result for this situation in the AGS. What is displayed is the measured rf frequency while the early flat top gymnastics occur including the bump pulsing. The bump has already started rising at the beginning of the trace. The bump peaks (800ms from T0, $100 \mathrm{~ms}$ on this trace) and then ramps back down - nearly down halfway across this trace. The vertical scale is frequency. The spacing between grid lines or label numbers is $5 \mathrm{~Hz}$. The total frequency change here as the bump ramps down is about $55 \mathrm{~Hz}$, close enough to our simple picture to be encouraging.

Comparing the bump time structure with the frequency picture shows a lot of correspondence though the frequency is not finished by $930 \mathrm{~ms}$. Perhaps the bump is really also not finished though the main current trace looks to be over.

This means for example that if the field were held fixed and the momentum (or velocity) were held fixed as the bump is energized then the frequency must decrease. The beam stays at the same radius outside the bump (momentum fixed) so that part of the orbit keeps the same path length. Inside the bump region the path length is increased. The total time to go around the ring increases. The frequency decreases.

Alternatively, if the frequency is held fixed (synchro on) and the magnetic field is at its original value with the bump up - when the beam extracts, then the momentum should change

For most of the analysis in this note we have only needed to assume that the field at a fixed time in the cycle doesn't change if we change the radial loop reference and hence the beam momentum. However, it is useful to further investigate whether the field has the same value with the bump up as it has with the bump down. In some of the following we will follow this path and see if this gives consistent beam behavior.

Two momentum-related experimental beam parameters available are the frequency and the average of the horizontal pues. The relation between frequency and momentum is complicated by the path length change introduced by the bump and by any transient field change due to the energizing of the bump magnets. The bump is created by powering AGS main magnet "backleg" windings. The bump power supplies necessarily couple into the main magnet power supply. Unlike most "historical" AGS high field backleg bumps, these bumps are constructed neither to keep any path length change zero to first order nor to keep any voltage introduced into the main magnet power supply zero to first order.

For simplicity we can assume that the magnetic field seen by the beam in magnets away from the bump is the same when we make our orbit measurements - at the bump peak and after the bump is back down, 800ms - 810ms and 1050ms - $1100 \mathrm{~ms}$. The bump changes the path length followed by the beam going around the AGS. We have a calculation from the Mad program for the increase in path length expected due to the bump if the momentum is kept fixed. Since keeping the momentum fixed keeps the beam at the same radius away from the bump, the calculated path length increase is local to the 
bump. For our standard bump (amplitude $\sim 50 \mathrm{~mm}$ to the outside at the kicker and at the septum) the path length is calculated to increase by $10.8 \mathrm{~mm}$.

Two simple situations can be analyzed. The magnetic field is assumed to be constant. Radius and frequency measurements are made bump on and bump off. In the first situation the momentum is assumed to be held fixed and the frequency change for the given path length change is calculated. In the second the frequency is held fixed and the momentum change required for the given path length change is calculated. If in fact the field is the same at both measurement times (of course the field can change between the measurements) and the radial loop holds the momentum constant, then both these situations can be realized in the AGS.

Situation 1: If the momentum or velocity is not changing, we expect a change in frequency $\mathrm{df} / \mathrm{f}$ equal to $\mathrm{dL} / \mathrm{L}$ the fractional path length change. Since $\mathrm{L}_{\mathrm{AGS}}$ (standard number $\mathrm{R}=128453 \mathrm{~mm} \times 2 \mathrm{p}$ ) $=807094 \mathrm{~mm}$, (and as stated above $\mathrm{dL}=10.8 \mathrm{~mm}$ ) then $\mathrm{df} / \mathrm{f}$ $=(10.8 / 807094)=1.34 \mathrm{e}-5$. Since Frf $=4.453720 \mathrm{Mhz}$, the predicted $\mathrm{df}=-59.6 \mathrm{~Hz}$.

Situation 2: holds the frequency constant (as does the synchro loop) and asks by how much the momentum changes. The radius at which the beam travels around the ring must decrease to compensate for the local path length increase within the bump. The momentum must decrease. One must be careful but the usual $(r, p, B)$ differentials apply. Bis fixed, $\mathrm{dr} / \mathrm{r}=\left(1 / ?_{\mathrm{tr}}{ }^{2}\right) \mathrm{dp} / \mathrm{p}$. The $(2 \mathrm{pdr})=-\mathrm{dL}$. From this $\mathrm{dr}=-1.72 \mathrm{~mm}, \mathrm{dr} / \mathrm{r}=-1.34 \mathrm{e}-$ 5 , and $\left(?_{\mathrm{tr}}=8.5\right) \mathrm{dp} / \mathrm{p}=-0.97 \mathrm{e}-3$.

The experimental numbers have been given in Appendix III. From Figure 2, the frequency change at fixed momentum ( situation 1) is $-62 \mathrm{~Hz}$ (vs $-59.6 \mathrm{~Hz}$ above). And the fixed frequency configuration (situation 2 ) gives from Table 1 , a change in $\langle\mathrm{r}\rangle \mathrm{p}$ by $2.08 \mathrm{~mm}$ (vs $-1.72 \mathrm{~mm}$ above) in the fixed frequency configuration ( situation 2 ).

The agreement is good, probably within errors, but how strongly does this result validate the assumptions made? How much field change is required to change the result by say $50 \%$ ? 